\title{
Personality and occupational stress in the field of mine rescue and intervention
}

\author{
Izabella Kovacs ${ }^{1, *}$, Artur George Găman ${ }^{1}$, Angelica Călămar ${ }^{1}$, Daniel Pupăzan ${ }^{1}$, and \\ Cristian Nicolescu ${ }^{1}$ \\ ${ }^{1}$ National Institute for Research and Development in Mine Safety and Protection to Explosion - \\ INSEMEX, 32-34 G-ral Vasile Milea Street, Petroșani, Hunedoara, Romania
}

\begin{abstract}
The special conditions created by emergency situations are likely to lead to psychological and emotional strains. For part of intervention and rescue personnel, these are accompanied by mobilization of internal resources, while in others these may lead to disabling phenomena as well as the occurrence of stress symptoms. Some people have a constitutional or psychological vulnerability to the action of traditional stressors, being more prone to psychic stress. The multifactorial complex of non-specific factors that modulate the individual response necessarily includes personality traits, acquired during the individual's experience and manifested in the context of its interactions with the social network, as well as in actual situations (stressful events). This paper presents the results of a series of psychological personality assessments of intervention and rescue personnel, in the context of analysing the relationship between personality traits and the risk of occupational stress occurrence.
\end{abstract}

\section{Introduction}

Performances of mine intervention and rescue personnel comes under the category "hard work", in terms of effort, multitude, diversity and especially nature of the tasks involved. Intervention and rescue personnel who, during or after intervention, experiences or is direct witness of violence, injuries, extreme destruction, long-time exposure to fatigue, severe weather, hunger, toxic contamination is at high risk for developing severe stress symptoms and problems related to long-term recovery.

Multitude, diversity and, above all, nature of tasks involved by rescue activities represent stressors that concur to exacerbate emotionality for those involved in this activity. Occupational risks and personal stressors are the core of vast majority of mine rescuer's stress reactions.

The manner in which intervention and rescue personnel deal with stressful situations depends on several variables. Personality characteristics, event circumstances, preparedness of the individual, pre-existing organizational stressors and pre-existing personal stressors are all key factors.

\footnotetext{
* Corresponding author: author@email.org
} 
The traumatic nature of an emotionally disturbing event is always the result of a subjective approach of this event by the individual and is not dependent only on objective characteristics of the event. If we consider stress-inducing events as capable of triggering stress in any individual, then we could witness a uniformity of behavioural reactions among people. However, a major feature of stressors is precisely their potential to produce stress as a result of the significance individuals assign them. In addition, same stressor cannot always cause stress in one and the same individual, on one hand because of current moods, as well as due to different meanings assigned to it at different times.

Thus, we can assert that a particular role in emergence and extent of psychic stress is played by cognitive, affective, motivational-aspirational and volitional features of the individual, shaped by his life experience and previous traumatic events. Peculiarities of personality, modelled by the individual's life experience, are involved in his response to a potential stressor, the significance that he assigns to stressors may lead to stress.

Some people have a mental, native or acquired vulnerability to stressors, being more prone to stress. Often, literature mentions the expression "vulnerability to stress", which in fact denotes an increased psychological receptivity to psychogenic stressors (and not only to them) capable of leading to mental stress [1]. Vulnerability to stress is accepted as a characteristic of some individuals to react easily through psychological stress to a wide range of stressors.

The following may be considered as indicators of vulnerability to stress [2]:

- interpretative tendencies on a difficult background;

- stiffness, stubbornness;

- egocentric, self-defence tendencies;

- obsessive and phobic tendencies on an anxious psychic background;

- impulsiveness, increased emotionality;

- aggression, inclination towards violence.

Vulnerability to stress builds up during life, depending on psycho-emotional trauma, experiences of severe psychological stressful situations, including how the individual succeeded in managing them. Analysis of different personality dimensions describes typologies more prone to psychological stress, such as: those with a high degree of introversion and / or neuroticism (Eysenck), those with mental stiffness (Kaln), those with low tolerance to frustration (Rosensweig), those whose intellectual affirmation or affiliation motivation is limited by adverse psychosocial factors [3].

\section{Stress and personality}

Stress represents a complex multidimensional process that includes:

- external or internal agent, called stressor / stress factor;

- evaluating and interpreting the stressor, assigning personal meanings;

- coping, the effort to dominate, reduce or ignore stressors;

- consequences that can be emotional, mental, physiological, behavioural.

Personality comprises many factors that, evaluated altogether, determine different types of psychological profiles. For any profile, it is possible to highlight aspects that protect the structure of personality in situations of distress, stress, ailment as well as fragility aspects that predispose personality to stress, manifestations such as malaise, disorders, anxiety and potential psychological decompensation.

Individual differences manifest themselves in all sequences of the stress process, namely exposure, evaluation, coping, short-term response and long-term consequences. These differences are mediators of the relationship between different daily situations and their consequences. Most obvious sources of individual differences may be categorised into three broad categories [4]: 
1. Genetic differences: physical, constitutional factors, reactivity, gender, etc.

2. Acquired demographic differences: education, profession, socio-economic status, marital status etc.

3. Personality factors or disposition variables: type A behaviour, negative affectivity that subsists anxiety, depression, neuroticism, self-beliefs, psychic strength (robustness), locus of control, flexibility, self-esteem, sense of consistency, self-esteem, self-image, responsiveness to experience, tolerance for ambiguity, aversion to risk, optimism, introversion - extraversion.

The personality characteristics of intervention personnel in toxic /flammable /explosive environments could help them cope with situations that general population would consider extreme. Personality traits involved in pathogenesis or sanogenesis can be divided into:

- Negative affectivity: depression, anxiety, neurosis;

- Positive affectivity or immunogenic features: optimism, humour, vitality, perceived efficacy, internal control, self-esteem (self-appreciation, selfconfidence).

Psychological examination appears as a prerequisite for proper management of occupational selection and psychological training of intervention and rescue personnel, for detailed assessment of personality and detection of those individuals who are vulnerable to stress. Psychological examination of employees should focus on both individual psychological diagnosis as well as individual prognosis. An important aspect of psychological assessment is to identify risk and protection factors associated with development and maintenance of emotional and behavioural problems. In this respect, within psychological examination, a particular role is played by personality assessment, both in terms of main personality traits that influence many types of behaviours as well as particular aspects, such as cognitive and behavioural coping with potentially stressful situations.

\section{Case study}

In a research carried out during 2018, at three economic agents in Jiu Valley, namely: Lonea Mine, Livezeni Mine and PrestServ, we aimed at assessing the impact that intervention and rescue operations had on rescuer's emotional state. Along with other aspects, the mentioned research assessed the personality of 42 mine rescuers, by using the following psychological instruments: NEO-PI-R personality inventory and SEC's attitudes and beliefs scale.

One of the most investigated personality traits in relation to stress is anxiety. Mechanisms by which anxiety influences vulnerability to stress occur both at assessment level and at response level. Anxiety is one of the faces of Neuroticism, along with angerhostility, depression, self-consciousness, impulsivity and vulnerability. High neuroticism or the tendency towards emotions and unpleasant feelings often interferes with the person's ability to adapt, positively correlates with the tendency to develop irrational ideas and with difficulties in managing stress. People with low anxiety and neuroticism scores are calm, able to cope with stressful situations without restlessness or exaltation. [11-14].

Personality tests mentioned above provide us with information about respondent's level of anxiety. As shown in figure 2, the vast majority of rescuers, subjects of the above mentioned research, have low and moderate levels of anxiety and, in general, medium levels of neuroticism (fig. 1.). The significance of these scores is that assessed persons are generally calm, able to cope with stressful situations, capable of adaptation and self-control.

Neurotic individuals evaluate stressors as more threatening than individuals with low scores on neuroticism, evaluate themselves, others and their own experiences in negative 
terms. High neuroticism is also associated with sense of inefficiency of coping strategies and longer lasting recovery after confrontation with stressful situations.

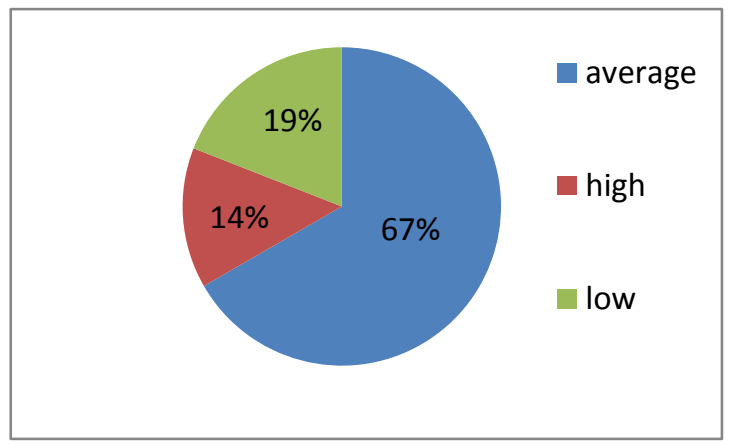

Fig. 1. Results for Neuroticism scale

A more general trait than anxiety is negative affection, defined as a sum of different disposition states: irritation, culpability, fear, depression, anxiety, neuroticism. Negative affection correlates in stressful situations with increases in catecholamine (hormones released in blood as a response to emotional and physical stress), cortisol and somatic symptoms [5]. Irritability as a trait, in that it causes increases in irritation in situations of frustration or conflict, is associated with marked increases in physiological activation. Along with hostility and aggression, irritability is considered a toxic factor for health and a good predictor for cardiovascular affections [4].

There are significant differences regarding the emotional scale among people with psychological or behavioural reactions to stress. Those reacting predominantly behaviourally exhibit more emotional instability, irritability, tension, are more impatient, anxious, sometimes even become angry. Those who react predominantly emotionally exhibit little impulsivity, calmness, patience [15]. Scores for Angry / Hostility (fig. 4.) scale place the respondents in the category of kind, rarely angry persons, the tendency to feel negative effects such as anger, bitterness and frustration being poorly present. Depression scale, at high and very high levels, indicates people prone to feeling guilt, feelings of helplessness, loneliness (fig. 4.). The type of behavioural activity correlates indirectly with depression, i.e. the ability of individuals to orient themselves towards purpose and to resolve stressful situations, increases with the decrease of pessimism, tension, indolence, dissatisfaction and irritability. Depressed people in stressful situations overstretch their energies, are unable to relax and work, give up rest and fun, are permanently strained and tensed while struggling to overcome situation, do things in hurry, have low frustration thresholds [15]

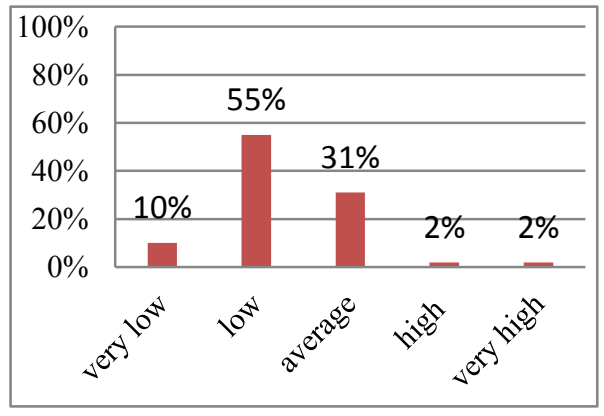

Fig. 2. Results for Anxiety scale

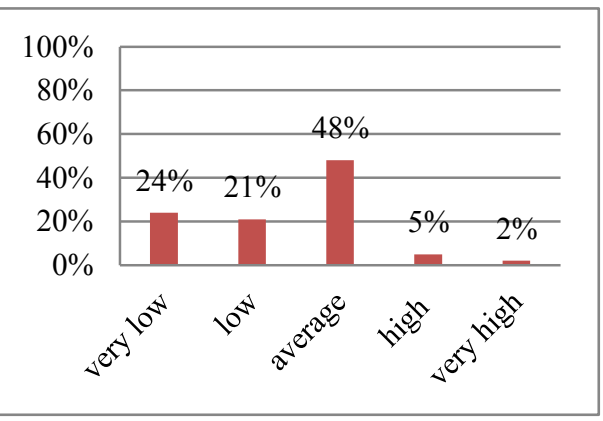

Fig. 3. Results for Angry Hostility scale 


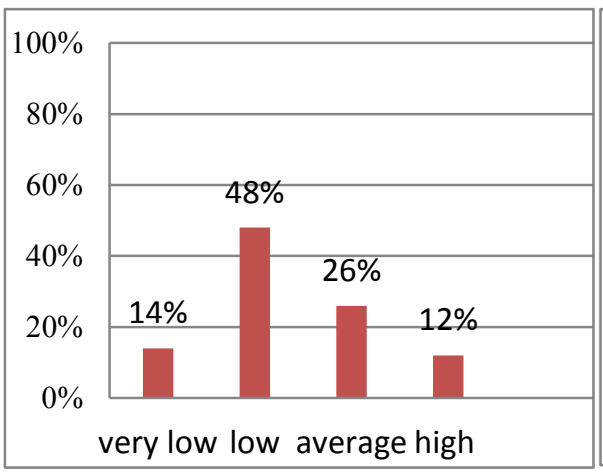

Fig. 4. Results for Depression Scale

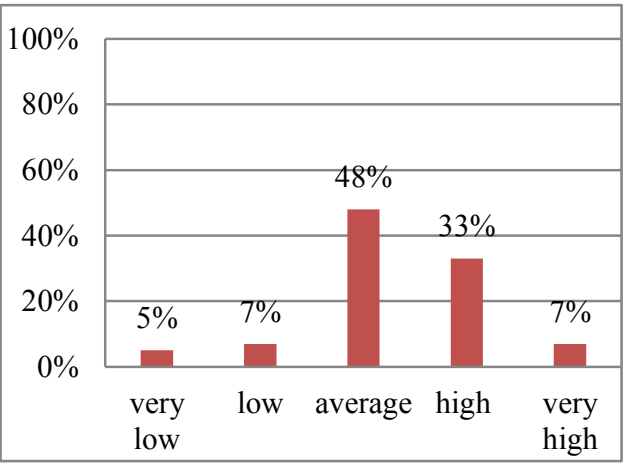

Fig. 5. Results for Self-Consciousness scale

Individuals with high scores on Self-Consciousness scale, as most of respondents in the research mentioned above (fig. 5.), do not feel at ease in the presence of others, feel inferior to those around them. Studies conducted [6] pointed out that negative affectivity (including Self-Consciousness) positively correlates with primary assessment of a situation's stress level and negatively with secondary evaluation of the effectiveness of coping strategies.

Neuroticism also comprises a facet called Vulnerability, which refers specifically to individual's vulnerability to stress. High and very high levels of Vulnerability (as shown by half of respondents in the research under consideration, (fig. 6.)) tell of people unable to successfully manage stress or cope with daily pressures and demands. These people become dependent, desperate and feel panic every time they find themselves in situations they consider to be problematic.

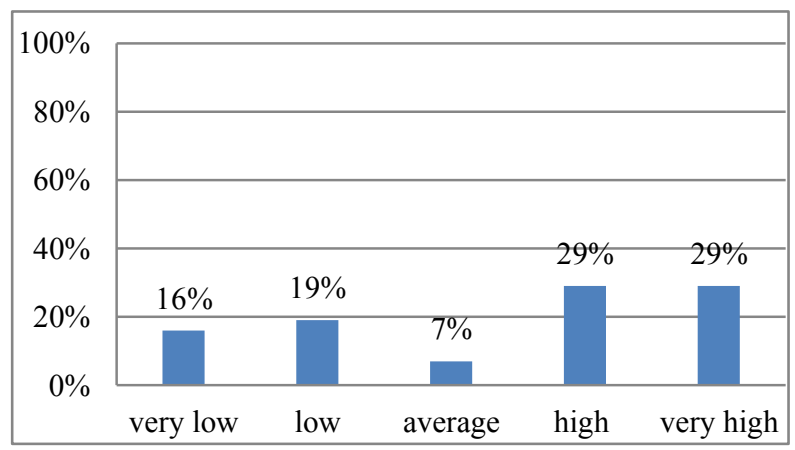

Fig. 6. Results for Vulnerability scale

Irrationality refers to assessments and beliefs that individuals have about themselves, world and life. At the root of emotional disturbances lies the individual's tendency to make unconditional and rigid evaluations of events. Results of a study conducted on Romanian population [16] revealed the following associations: irrational beliefs are positively associated with emotional distress, other cognitive distortions, anxiety and depression (assessed as complex cognitive, behavioural, physiological and subjective manifestations). Also, irrational beliefs are negatively associated with unconditional acceptance of one's own person.

Low, very low levels, and under certain conditions, even average irrationality tells of people that have non absolute personal beliefs, leading to positive/negative but adaptive and flexible emotions, without interfering with reaching for individual or group purposes. This situation is found in one third of respondents in the research under consideration (fig. 7.). 
High and very high levels of irrationality (found in almost $60 \%$ of participants, fig. 7 ) are associated with disabling cognitive structures, dysfunctional negative emotions generating emotional distress, depression and anxiety. These people have a low level of unconditional self-acceptance, which represents a protective factor in dealing with negative life situations.

Fig.7. Results for Irrationality

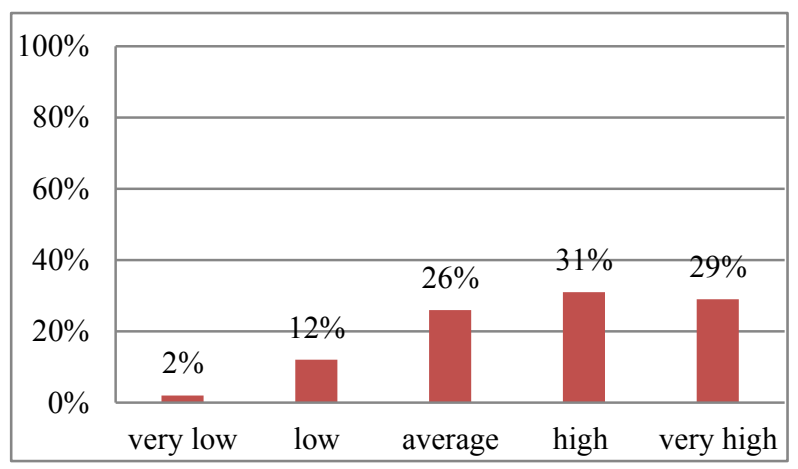

As mentioned before, according to literature, individuals who achieve high levels of stress are those who exhibit rigidity and aggressiveness, but also self-centeredness and social disengagement are highly correlated with stress $[7,12,14]$.

Stress factors are perceived as more or less threatening, stress reactions are triggered or not depending on the degree to which the person is able to activate coping mechanisms. Choosing a strategy or another, preference for direct action versus activation of defensive mechanisms is influenced by individual differences.

Most common and often used classification of coping is:

(1) coping focused on problem / task (development of plans and involvement in actions to directly respond to stressors);

(2) emotion-oriented coping (involves attempts to adjust emotional response to problem);

(3) assessment-oriented coping (which refers to redefining stressful situation in more acceptable terms).

Some authors have assumed a negative relationship between problem-oriented coping and distress and a positive relationship between emotion and distress, considering direct confrontation with the stressor (problem orientation) as beneficial opposed to efforts to focus on emotional response, which is a loss of energy because it focuses on symptoms, not the cause. Research results were contradictory for both hypotheses, which led to suggesting a contingent approach regarding the use and effectiveness of coping strategies. The arguments used were that the use of emotion-oriented coping in a changing situation or problem-oriented coping in a situation that cannot be changed increases the distress because of the mismatch between the choice of strategy and its efficiency. In this context, what is most important is the individual's focus on identifying a strategy that promises efficiency [8]. Also, active and pro-social coping strategies have tended to associate more often with positive emotional consequences, with low levels of anxiety and depression, while antisocial coping has been associated with increased levels of anger, and avoidance strategies with an increased level of anxiety and depression. [9]

Sociable people, confident in their interpersonal skills, use more often direct coping actions compared to avoidance mechanisms [4]. Agreeableness is the personality factor that relates to interpersonal tendencies. High levels of Agreeableness are characteristic of sympathetic, self-serving individuals, willing to help others and confident that others will also help them. 


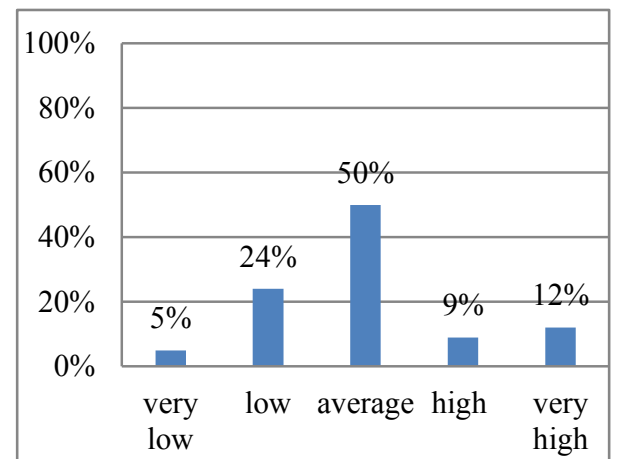

Fig. 8. Results for Altruism scale

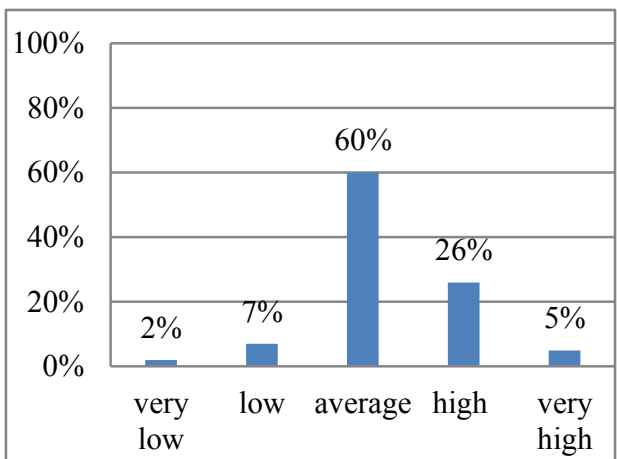

Fig. 9. Results for Trust scale

High levels of stress have been identified in people with low scores at social presence (expressed as reserved, not expressing opinion, prudent). Significant stress-related symptoms are also seen in people with low confidence, those with low independence, slightly irritated, and angry, who insist on doing things as they think, even if this causes conflicts (low good impression). Also, high levels of stress are recorded by people who have low scores on tolerance (lack of trust in people, criticizing other's opinion, selfish, hostile, and vengeful), empathy (indifferent, lacking motivation, preoccupied with what people do rather than what they feel or think), as well as on work orientation (unstable people, slightly distracted from priority tasks, with fluctuations of disposition) [12].

A quarter of respondents achieved low scores on altruism scale (fig. 8.), which places them in the area of self-centred people, centred on their own interests and desires and too unwilling to get involved in problems of others. In terms of trust (fig. 9.), the general tendency of respondents is to think that those around them are well-intentioned, honest, considering themselves as open and sincere.

Regarding another major personality dimension, namely conscientiousness, it has been identified as a powerful predictor of problem-based adaptation strategy, such as planning, finding appropriate solutions, and positively assessing events. The explanation is that people motivated to overcome the obstacles they face, who are organized and have a sense of resentment in their own actions, tend to try to master stressful situations rather than to anchor in negative emotions or avoid them $[10,12]$.

There are studies according to which it is less likely that the very conscientious will experience stress during periods of professional training. However, other studies do not report significant correlations between consciousness and stress levels [7].

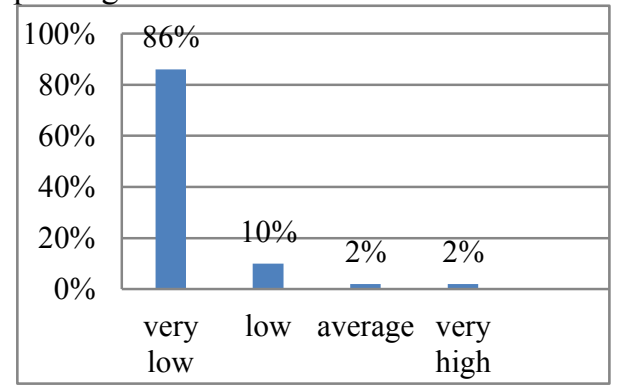

Fig. 10. Results for Self Discipline scale

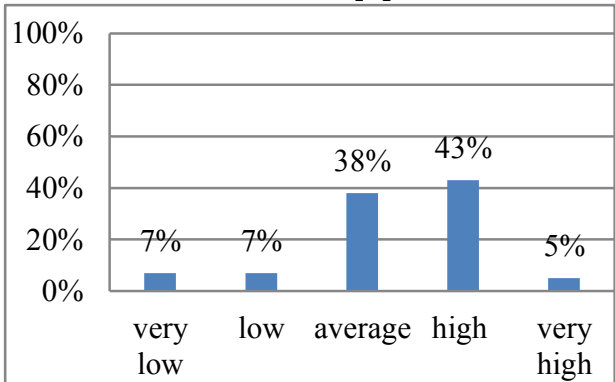

Fig. 11. Results for Dutifulness scale

Scores achieved by respondents at two faces of Conscientiousness, namely Selfdiscipline and Dutifulness are located at opposite poles. While the Dutifulness is rather present (fig. 11.), not the same can be said about Self-discipline (fig. 10.). This may 
translate into the fact that we are dealing with people who, although generally adhere to their ethical principles and fulfil their moral duties, are mainly characterized by a lack of motivation, a tendency to easily be discouraged, not to persevere in the task, to be easily distracted from their purpose. According to studies mentioned above, these characteristics of personality predispose the individual to high levels of stress.

Resistance and psychological resilience of individuals can be improved, thus increasing their chances of dealing with stressful events and their consequences.

Resistance refers to an individual's ability to withstand clinical manifestations of distress or dysfunction associated with critical events, and can be seen as a form of psychological-behavioural immunity.

Resistance can be increased by the following: realistic training (building adequate expectations, developing coping abilities and stress management); development of group cohesion and social support; developing positive thinking and developing the sense of selfefficacy.

Resilience refers to an individual's ability to adapt, to quickly and efficiently return from psychological / behavioural disturbances associated with critical events. Resilience is an ability of those able to withstand difficult situations. Genes only account for $50 \%$ of a person's response. Another $10 \%$ is circumstance-based, and $40 \%$ is "intentional activity", which we can influence through behaviour [17]. Critical psychological elements and resilience characteristics are: optimism (those who are extremely optimistic tend to show greater resilience), altruism, faith and spirituality, humour, having a role model, social support (contact with others who can be trusted, family, friends), confront fears, have a mission in life and exercise (helps improve brain functions and develop the discipline necessary for perseverance).

\section{Conclusions}

The multitude, diversity and, above all, nature of tasks that rescue work entails are stressors that contribute to exacerbating emotionality for those involved in rescue activities.

All personality peculiarities, modelled by the individual's biography, are involved in his response to a potential psychic stressor, contributing to conferring it a noxious, imaginary or real significance, capable of leading to stress.

Vulnerability to stress builds up during the individual's life, depending on psychoemotional trauma, experiences of severe psychological stressful situations, including how he managed to cope. In terms of stress, there are a number of personality traits involved in pathogenesis or sanogenesis, namely negative affectivity (depression, anxiety, neuroticism) and positive affectivity or immunogenic features (optimism, humour, vitality, perceived efficacy, internal control, esteem self).

Because overall stress cannot be eliminated, the primary goal in coping with stress is to feel positive stress rather than distress and prevent distress to degenerate into dysfunctional behaviours. In the context of intervention and rescue activities, dysfunctional behaviours such as altering decision-making, mistakes, impulsive and disorganized behaviour, behavioural paralysis or panic can result in additional damage and loss of human life.

The results of NEO-PI-R and SEC tests, on a number of 42 mine rescuers, indicate that the referred to intervention and rescue personnel shows highly developed immunogenic and disimmunogenic (whose presence is associated with emotional and even psychopathological problems) personality traits.

Resistance and resilience to stress can be enhanced by specific actions that can be integrated into training programs for mine rescue personnel. 


\section{References}

1. I.B. Iamandescu, Medical psychology (Infomedica Pub. House, Bucharest, 2005)

2. C. Borangic, Somatotherapy Journal 12 (2007)

3. I.B. Iamandescu, Stress and internal diseases (All Pub. House, Bucharest 1993)

4. A. Băban, Stress and personality (Presa Universitară Clujeană Pub. House 1998)

5. A. I. Saeed, The influence of neuroticism on stress perception and its resultant negative affect, Master's Theses 3965 http://scholarworks.sjsu.edu/etd_theses/3965 (2011)

6. D. David, Clinical assessment system (Romanian Psychological Testing Services, Cluj Napoca, 2007)

7. B. Balgiu, Romanian Medical Journal LVI, 3, 214-217 (2009)

8. A. T. Brațe, Aggressiveness and Organizational Stress: Models, Diagnosis and Management (University Publishing, 2014)

9. A. Malkoç, Procedia Social and Behavioural Sciences 12, 426-435 (2011)

10. O. Budău, SACS: the strategic coping approach scale (ASCR Publishing, Cluj-Napoca, 2010)

11. V. M Dumitru, D Cozman, HVM Bioflux 4, 1, 34-39 (2012)

12. V. Robu, Personality and styles to adapt to professional stress. The results of a military cadre study in Psychology of the Military Organization and the Psychological Selection of the Human Resource (Carol I National Defence University Publishing, Bucharest, 2007)

13. P. G. Leandro, D. Castillo, Procedia Social and Behavioural Sciences 5, 1562-1573 (2010)

14. A. Karimzade, M.A. Besharat, Social and Behavioural Sciences 30, 797-802 (2011)

15. R. McCrae, P.T. Costa, Personality in adulthood (The Guilford Press, New York, 1990)

16. E. Losii, Influence of personality traits on the type of reaction in occupational stress, Psychology, Special Pedagogy, Social Assistance 18 (2010)

17. P. Jeffrey, Power: Why Some People Have It, and Others Don't (HarperCollins, New York, 2010) 